\title{
Accounting for the Dependence of Coil Sensitivity on Sample Thickness and Lift-Off in Inductively Coupled Photoconductance Measurements
}

\author{
Lachlan E. Black ${ }^{(1)}$ and Daniel H. Macdonald ${ }^{(1)}$
}

\begin{abstract}
Inductively coupled photoconductance measurements are widely used to characterize carrier recombination in crystalline silicon. We show that, contrary to what is usually supposed, the sensitivity of such measurements is significantly dependent on sample thickness in the range of typical wafer thicknesses, due to the attenuation of the magnetic field with distance from the coil. Sample thickness, as well as any separation from the coil, should, therefore, be taken into account in system calibration in order to avoid systematic errors. We investigate the magnitude of this effect both experimentally and via analytical and finite-element modeling for a range of commercial photoconductance measurement systems with varying coil geometry. Finite-element modeling is used to identify the functional form of the attenuation in the regime of interest, and simple formulae are derived which allow the experimentalist to correct for sample thickness and lift-off. Close agreement is found between modeled and experimental attenuation behavior. Finite-element modeling is also used to evaluate the magnitude of skin effects, which are found to have a minor influence on the measured conductance for the most highly conductive samples, and to determine the lateral spatial variation of the coil sensitivity, which is important for lifetime imaging techniques where photoconductance measurements are used for calibration.
\end{abstract}

Index Terms - Charge carrier density, charge carrier lifetime, conductivity measurement, eddy currents, measurement techniques, photoconductivity, photovoltaic cells, silicon.

\section{INTRODUCTION}

$\mathbf{C}$ ONTACTLESS, inductively coupled photoconductance decay measurements are a standard, widely used tool for characterizing carrier recombination in crystalline silicon [1]-[5]. Such measurements utilize the principle of eddy current detection to measure the free-carrier conductivity (due to, e.g., doping or optical excitation) of a silicon wafer or ingot in proximity to an inductive coil. An alternating current passed through

Manuscript received July 5, 2019; revised September 6, 2019; accepted September 14, 2019. Date of publication October 18, 2019; date of current version October 28, 2019. The work of L. E. Black was supported by the Australian Renewable Energy Agency under Project RND017. (Corresponding author: Lachlan E. Black.)

L. E. Black is with the Research School of Electrical, Energy and Materials Engineering, The Australian National University, Canberra, ACT 2600, Australia, and was previously with the Department of Applied Physics, Eindhoven University of Technology, 5612 AZ Eindhoven, Netherlands (e-mail: lachlan.black@anu.edu.au).

D. H. Macdonald is with the Research School of Electrical, Energy and Materials Engineering, The Australian National University, Canberra, ACT 2600, Australia (e-mail: daniel.macdonald@anu.edu.au).

Color versions of one or more of the figures in this article are available online at http://ieeexplore.ieee.org.

Digital Object Identifier 10.1109/JPHOTOV.2019.2942484 the coil generates a magnetic field, which induces an opposing current flow and associated magnetic field in the sample. This has the effect of increasing the impedance of the coil, resulting in a voltage drop across the coil which is approximately proportional to the sample conductance. The absolute relationship between coil voltage and sample conductance can be determined by calibrating against samples of known conductance (e.g., differently doped samples where the conductance has been measured by four-point probe), and absolute conductance can be converted into carrier concentrations through a knowledge of the carrier mobilities. Thus, such measurements can be used to monitor the excess carrier population as a function of time under optical excitation, from which the carrier recombination lifetime may be inferred [1]-[5].

It is understood that the sensitivity of such a coil drops off with distance, such that a smaller voltage is measured for conductive material located further from the coil. This has obvious implications for the measurement of very thick samples (such as ingots) and of samples separated from the coil by a finite "liftoff" distance, as commonly required when using wet-chemical solutions to passivate wafer surfaces [6]. These cases have been the subject of work by Swirhun et al. [7] and Favre et al. [8] respectively. Swirhun et al. [7] characterized this effect for the Sinton Instruments BLS-I bulk lifetime tester system both by comparing measurements of wafers and bulk samples of various conductivities and by measuring the response to a conductive wafer offset to different distances from the coil using undoped spacer wafers. They reported an exponential decay in sensitivity with distance, with a characteristic depth equal to approximately $2.5 \mathrm{~mm}$. Favre et al. [8] reported a similar experiment using spacer layers and variously doped wafers to characterize the influence of coil-to-sample lift-off for the Sinton Instruments WCT-120 system, which is the system most commonly used to characterize wafer samples. They also used an exponential decay function to describe their data, finding a characteristic depth of approximately $3.2 \mathrm{~mm}$.

What is perhaps less obvious and has so far received little attention is the fact that coil sensitivity attenuation is also of importance even when measuring thin samples (i.e., wafers) placed directly on the coil, if these samples are of different thickness to those used for calibration. For instance, the standard calibration wafers supplied by Sinton Instruments are approximately $525 \mu \mathrm{m}$ thick, but measured sample wafers are commonly as thin as $180 \mu \mathrm{m}$ or less. As we have recently shown for the 
Sinton Instruments WCT-120TS system, such a difference in thickness can result in a relative difference in sensitivity of up to $10 \%$, and a resulting overestimation of the conductance by the same amount [9]. While this may not seem very large, it can still result in errors that are significant for certain applications. This is particularly the case when one is interested in lifetime behavior in high-injection, since the lifetime in this regime is strongly dependent on the carrier concentration. Our initial investigation of this effect showed how measurements of calibration samples with a range of thicknesses, and stacked in different configurations, could be used to experimentally determine the characteristic attenuation length of a particular system, in good agreement with values predicted by numerical modeling [9].

In this article, we extend our investigation of this effect to a wider range of inductively coupled photoconductance lifetime tester systems, including the most commonly used Sinton Instruments WCT-120 model, as well as the WCT-120PL, WCT120TS, and a custom-built small-coil system. Finite-element modeling is first validated against established analytical solutions for the case of a coil in free space, and then used to identify the functional form and magnitude of the attenuation as a function of sample thickness and lift-off for the actual system geometries. The characteristic attenuation lengths derived from finite-element modeling of each system are then compared to experimental values determined by applying the attenuation functions to correct measured calibration data. Individual examples of each system at several different laboratories are characterized in order to establish the magnitude of system-to-system variability. In addition to the influence of sample thickness, we systematically investigate the dependence of sensitivity on sample lift-off both for uniformly doped samples of finite thickness and for thin conductive sheets (e.g., diffused surfaces). Measurements of single-side-diffused wafers separated from the coil by spacer layers of variable thickness are used to directly measure the attenuation of coil sensitivity as a function of lift-off, in order to confirm the functional dependence predicted by modeling. Finite-element modeling is also used to assess the importance of skin effects and to determine the radial variation of the coil sensitivity, which is important for imaging applications.

\section{EXPERIMENTAL DETAILS}

Inductively coupled conductance measurements were performed using various commercial photoconductance lifetime measurement systems located at different laboratories. All were built by Sinton Instruments. They included examples of the WCT-120, WCT-120PL, and WCT-120TS models, as well as a custom-built small-coil system designed for measuring small samples. The measured quantity in all cases is the voltage drop across an inductive coil connected to an ac $(13.56 \mathrm{MHz})$ current source. In all cases, the coil consists of three turns of $0.66 \mathrm{~mm}$ diameter wire and is located at the center of a cylindrical cavity bounded by a metal field plate, which extends under the sample stage outside the coil cavity. The different systems differ in the diameters of the coil $\left(\oslash_{\text {coil }}\right)$ and field plate $\left(\oslash_{\text {plate }}\right)$, as well as the thickness and type of (nonconductive) casing present between the coil and the sample. Sample voltages $\left(V_{\text {wafer }}\right)$ were measured in the dark and compared with measurements of the voltage without a sample present $\left(V_{\text {air }}\right)$ taken immediately prior.
As samples, we used two sets of 4 in diameter, bulk-doped, (100) silicon wafers of varying resistivity and thickness, intended as photoconductance calibration samples. One calibration sample set (Set \#1) was prepared and characterized at the Eindhoven University of Technology, while a second, similar calibration set (Set \#2) was subsequently prepared and characterized independently at the Australian National University (ANU). Comparison measurements of the two sample sets performed using the same photoconductance measurement system showed excellent agreement between the conductance-voltage relationships obtained for the two datasets.

Some of the samples in each set were single-side polished calibration wafers supplied by Sinton Instruments. Others were double-side polished wafers acquired commercially. All had a nominal thickness of approximately $525 \mu \mathrm{m}$ as-supplied. Several sister wafers with sheet conductance corresponding to the upper end of the instrument sensitivity range were thinned down uniformly by etching in either $\mathrm{KOH}$ or tetramethylammonium hydroxide (TMAH) solution to give similarly doped samples with a range of thicknesses.

The sheet conductance of all wafers was determined by the four-point probe method using the slope of the voltage-current relationship measured by a Keithley 2425 SourceMeter in the dark. A thermocouple connected to the measurement stage was used to monitor the temperature during the measurement. Current ranges were chosen to give a measured voltage range of approximately $\pm 10 \mathrm{mV}$ for each sample. Thickness correction factors for four-point probe measurements were calculated using Weller [10, eq. 24] (we note that commonly cited approximate expressions for the thickness correction factor given in [11], and originating from the work of [12], can result in significant errors - up to $\sim 8 \%$-in the range of typical Si wafer thicknesses, depending on probe spacing). Wafer thickness was measured using a contact dial gauge system with digital readout and $1 \mu \mathrm{m}$ resolution. Both sheet conductance and thickness measurements were performed at at least five different points in the vicinity of the region measured by the coil, and averaged to give final values. The relative standard deviation of the measured thickness was always less than $1 \%$ and generally less than $0.6 \%$, while the relative standard deviation of the measured conductance was always less than $1.6 \%$ and generally less than $0.7 \%$. The measured sheet conductances were corrected for temperature differences between the four-point probe and inductively coupled conductance measurements according to the mobility model of [13] and [14].

Additional single-side-diffused samples were prepared on high resistivity $(\geqslant 100 \Omega \mathrm{cm})$ p-type $\mathrm{Si}$ wafers. A heavy n-type phosphorus diffusion was formed on both sides of the wafers in a diffusion furnace using a $\mathrm{POCl}_{3}$ source and driven in to give a highly conductive $(\sim 6.66 \Omega / \square) n+$ sheet diffusion. The phosphosilicate glass layers were subsequently removed by etching in HF, and the diffused region etched away on one side to leave single-side-diffused samples.

\section{MODELING}

The problem of the eddy current distribution induced by an inductive coil in an infinite plane conductor of finite thickness 
and finite separation from the coil (lift-off) was considered in the seminal work of Dodd and Deeds [15], who derived analytical solutions for the axially symmetric case, where the coil is circular with a rectangular cross section and its axis aligned normal to the conductive layer. These expressions can be used to calculate the change in the impedance of the coil as a function of sample thickness, lift-off, and conductivity.

In the actual measurement systems, the coil is not in free space, but is located in a circular cavity bounded by a metal field plate with a finite diameter. In this situation, the treatment of Dodd and Deeds is not applicable. Therefore, in order to evaluate the effect of this feature, finite-element modeling was performed using the Finite Element Method Magnetics software [16], assuming a 2-D, axisymmetric geometry. The individual turns of the coil were modeled as circular cross sections. The top of the coil was assumed to be level with the top of the field plate, which was assumed to extend to a depth of $1 \mathrm{~cm}$ below the plane of the sample stage. The sample was separated from the coil and the field plate by a gap of $10 \mu \mathrm{m}$ at a distance defined as $z=0$. The relative magnetic permeability of the sample was assumed to be equal to 1 .

The software yields the coil impedance directly as an output of the simulations. The relative coil sensitivity as a function of sample thickness and lift-off was determined from the change in the real part of the coil impedance relative to the case when no sample was present, divided by the sample thickness and conductivity, and normalized to the value for an infinitesimally thin sample positioned at zero separation from the coil. The modeled conductivity was kept low enough to make skin effects (as discussed in Section IV-C) insignificant.

\section{A. Validation of the Numerical Model}

In order to validate the numerical model, we first compare its results to those of the analytical model of Dodd and Deeds [15] for the case when the field plate is not present (i.e., the coil is in free space). Fig. 1 shows the simulated vector potential distribution and magnetic flux for a coil geometry corresponding to that of the WCT-120 system $\left(\oslash_{\text {coil }}=18.74 \mathrm{~mm}\right)$ both with and without the inclusion of the field plate. Fig. 2 shows the corresponding relative sensitivity as a function of sample lift-off for both cases, as well as according to the analytical model. In the analytical model, the sample was infinitesimally thin, while in the numerical model the sample was $10 \mu \mathrm{m}$ thick. We obtain very good agreement between the analytical and numerical models for the case when no field plate is present. The slightly higher relative sensitivity predicted by the analytical model at larger lift-off values is due to the fact that the analytical model assumes a sample of infinite diameter, while in the numerical modeling the sample diameter was limited to $10 \mathrm{~cm}$. Note that in the analytical model, the coil was treated as having a rectangular cross section with width equal to the wire diameter and height equal to the product of the diameter and the number of turns, while in the numerical model, the individual turns of the coil were modeled with circular cross sections. However, this difference was found to have minimal impact on the results.
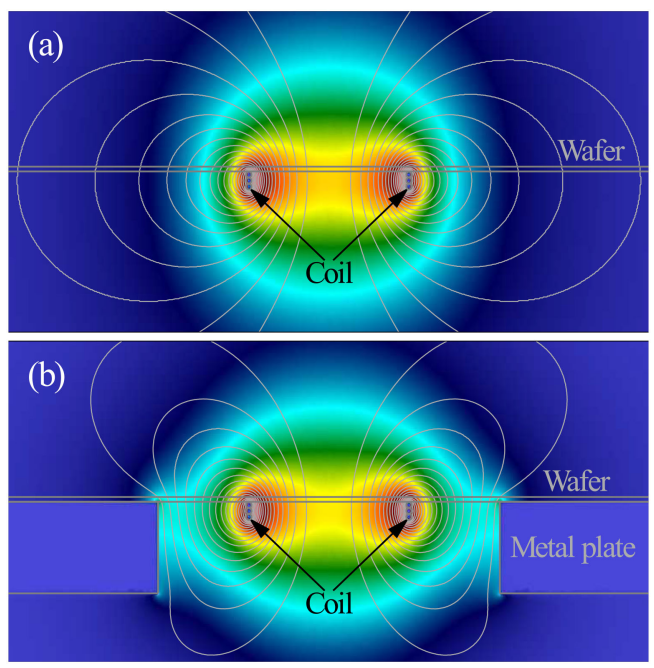

Fig. 1. Cross-sectional view of the WCT-120 coil, (a) in free space and (b) with the metal field plate, showing the simulated vector potential distribution (contours) and magnetic flux density (density gradient) for the case when no conductive sample is present (the presence of a typical silicon wafer sample has only a minor effect on the qualitative potential distribution). The position of the sample wafer relative to the coil is indicated for clarity.

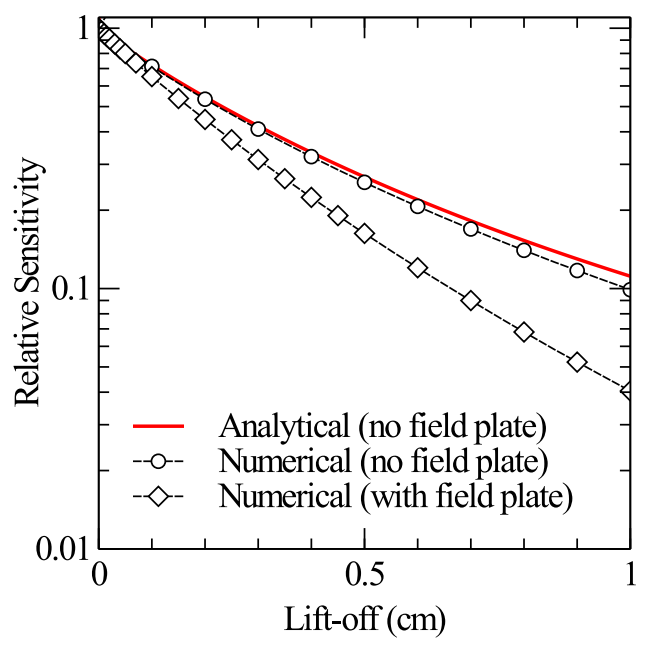

Fig. 2. Relative sensitivity of the WCT-120 coil as a function of sample lift-off, calculated either according to the analytical model of Dodd and Deeds [15] or from numerical modeling with or without the inclusion of the field plate.

\section{B. Effect of System Geometry}

Fig. 2 also shows that the inclusion of the field plate in the modeling results in a significantly more rapid attenuation of the sensitivity with distance from the coil. This can be understood intuitively as a consequence of the initial constriction of the magnetic field within the cavity bounded by the plate, as shown in Fig. 1(b). Reducing the diameter of the cavity enhances this effect.

Fig. 3 compares the numerically modeled sensitivity attenuation as a function of lift-off for the different system geometries investigated. As expected, the attenuation predicted for the WCT-120TS geometry, with its smaller diameter coil and field plate, is significantly more rapid than for the WCT-120/WCT$120 \mathrm{PL}$, with a $60 \%$ greater reduction in relative sensitivity at 


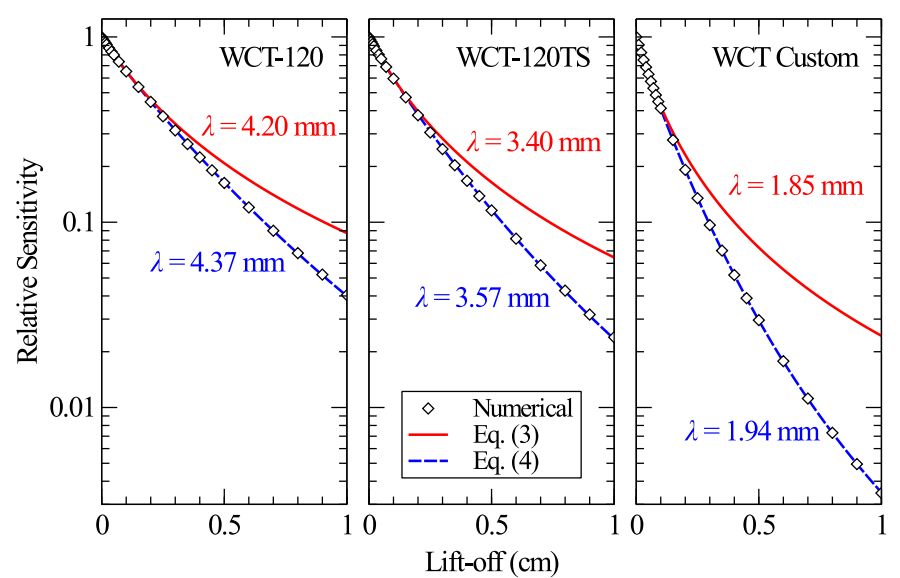

Fig. 3. Relative sensitivity of the coil as a function of sample lift-off calculated from numerical modeling for the WCT-120, WCT-120TS, and WCT Custom systems. Lines show least squares fits using (3) for data between 0 and $1 \mathrm{~mm}$, and using (4) for data between 0 and $10 \mathrm{~mm}$, where labels show the value of $\lambda$ corresponding to the fit.

$1 \mathrm{~cm}$ lift-off. The rate of attenuation is even more rapid for the very small coil of the WCT Custom system, with a more than ten times greater reduction in relative sensitivity at $1 \mathrm{~cm}$ lift-off.

\section{Functional Description of the Attenuation}

It is apparent from the semilogarithmic plots in Figs. 2 and 3 that the functional form of the sensitivity attenuation may only crudely be described as exponential. Indeed there is no physical reason to expect exponential behavior in this case. The actual behavior is mathematically rather complicated, as may be inferred from the complexity of the equations which arise in the analytical treatment of Dodd and Deeds, and it is not easy to find a simple single-parameter function which provides a good description over the whole distance range to $1 \mathrm{~cm}$. Depending on the domain of interest, different functions provide a better or worse approximation. Since we are primarily concerned here with the effect of sample thickness, we start by considering the domain between 0 and $1 \mathrm{~mm}$, which covers the range of typical wafer thicknesses, and rather than the attenuation function itself, we consider the integral of this function between 0 and $W$, where $W$ is the sample thickness.

Fig. 4 shows the numerically modeled relative sensitivity versus sample thickness for the different system geometries between 0 and $2 \mathrm{~mm}$. Again, it is clear that the attenuation becomes more rapid as the diameters of the coil and field plate decrease. We find that a simple, single-parameter empirical expression that provides a very good fit of the modeled values in this range is

$$
S(W)=(1+W / \lambda)^{-1} .
$$

This expression is able to fit the modeled values with a maximum relative error of less than $0.1 \%$ between 0 and $1 \mathrm{~mm}$ for the WCT-120 and WCT-120TS systems, and less than $0.6 \%$ for the small-coil WCT Custom system. The error increases at larger thicknesses, but is still only around $1 \%$ at a thickness of $2 \mathrm{~mm}$ for the WCT-120 and WCT-120TS systems.

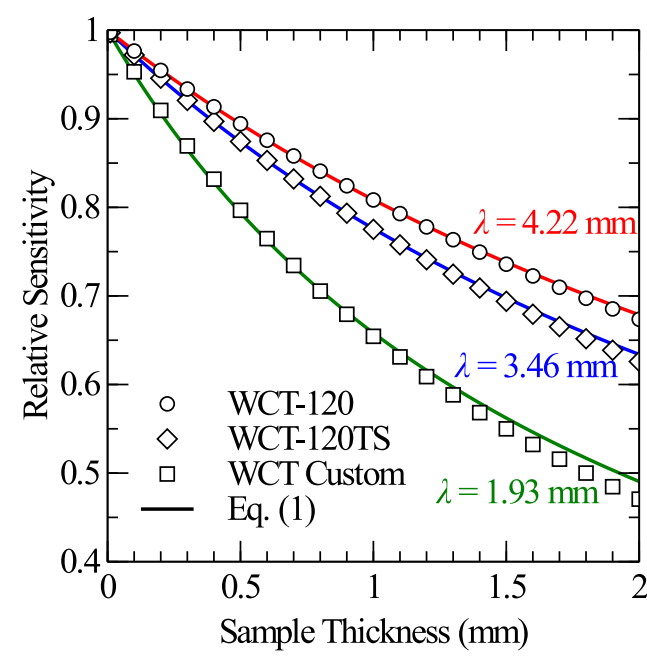

Fig. 4. Relative sensitivity as a function of sample thickness calculated from numerical modeling of the different photoconductance system geometries. Lines are fits of (1) to the modeled values between 0 and $1 \mathrm{~mm}$, where labels show the value of $\lambda$ corresponding to the fit.

From (1), it follows that the relative sensitivity factor for a sample of uniform conductance and thickness $W$, separated from the coil by a lift-off distance $\Delta z$, is given by

$$
S(W, \Delta z)=(1+\Delta z / \lambda)^{-1}(1+(W+\Delta z) / \lambda)^{-1} .
$$

Similarly, the relative sensitivity for a conductive sheet at a distance $z$ from the coil is given by the derivative of $z S(z)$, which we designate $S^{\prime}(z)$, which is simply

$$
S^{\prime}(z)=(1+z / \lambda)^{-2} .
$$

Fig. 3 shows that (3) describes the modeled sensitivity as a function of lift-off well in the region close to the coil.

\section{Attenuation Functions Valid at Larger Distances}

Equations (1) and (3) constitute good approximations for $W$ or $z$ up to about $\lambda / 3$. Beyond this point they systematically overestimate the sensitivity. Empirically, we find that the deviation of (3) from the data with increasing thickness can be compensated to a good approximation by the addition of a second multiplicative term, which is also a function of $\lambda$. The resulting refined expression for $S^{\prime}(z)$ is given by

$$
S^{\prime}(z)=(1+z / \lambda)^{-2}\left[1+(z / 2 \lambda)^{2}\right]^{-1} .
$$

Fig. 3 shows that (4) can be used to describe the modeled sensitivity as a function of lift-off with good accuracy even up to $1 \mathrm{~cm}$.

An analogous simple expression for $S(W)$ or $S(W, \Delta z)$ valid at greater distances from the coil is less easy to find. However, $S(W, \Delta z)$ can be calculated either analytically or numerically from (4) using the general relationship

$$
S(W, \Delta z)=W^{-1} \int_{\Delta z}^{\Delta z+W} S^{\prime}(z) \mathrm{d} z .
$$

For most practical applications we will consider, (1)-(3) are sufficiently accurate. Equation (4) will become important only 
when $\Delta z+W>\lambda / 3$, which for most systems is likely to be the case only when the sample lift-off is large (e.g., when measuring samples separated from the coil by a petri dish or other thick spacer layer).

\section{E. Correcting Measured Data}

The effective conductance seen by the coil is equal to the product of the sample conductance and the relative sensitivity. For a uniformly conductive sample (e.g., a uniformly doped semiconductor wafer), the relative sensitivity is given by $S(W, \Delta z)$. For a sample with one or more highly conductive surface regions much thinner than the sample thickness $W$ (e.g., a wafer with a diffused or implanted surface dopant profile, or a thin highly doped epitaxial layer), the relative sensitivity of the coil to these surface regions is given by $S^{\prime}(z)$, where $z$ is the distance of the surface from the coil. Thus, in the case of a wafer sample with a uniformly conductive bulk, and highly conductive surface regions on both sides, the total effective conductance $\sigma_{\text {eff }}$ seen by the coil is given by

$$
\begin{aligned}
\sigma_{\text {eff }}= & S(W, \Delta z) W \rho_{\text {bulk }}^{-1} \\
& +S^{\prime}(\Delta z) R_{\text {sheet }, 1}^{-1}+S^{\prime}(\Delta z+W) R_{\text {sheet }, 2}^{-1}
\end{aligned}
$$

where $\rho_{\text {bulk }}$ is the bulk resistivity of the sample, and $R_{\text {sheet }, 1}$ and $R_{\text {sheet, } 2}$ are the sheet resistances of the highly conductive surface regions closest to and furthest from the coil, respectively.

In the most general case, where the sample conductivity is a function of depth in the sample, we have

$$
\sigma_{\text {eff }}=W^{-1} \int_{\Delta z}^{\Delta z+W} S^{\prime}(z) \sigma_{z}(z) \mathrm{d} z
$$

where $\sigma_{z}(z)$ is the depth-dependent sample conductivity. The use of (7) may be necessary, for example, in the case of a significantly nonuniform excess carrier profile, as can occur when surface recombination is large or excitation is nonuniform over the sample depth.

\section{RESULTS AND DISCUSSION}

\section{A. System Dependence of the Attenuation Length}

As we showed in our initial investigation [9], the characteristic attenuation length $\lambda$ can be experimentally determined for a given system by applying (6) to correct the sample conductance in measurements of calibration samples with a range of thicknesses and/or stacked in different configurations. In this procedure, $\sigma_{\text {eff }}$ calculated from the independently measured sample conductance using (6) is plotted against the coil voltage measured for the same samples, as in a standard calibration measurement. $\lambda$ is treated as a free parameter, and chosen so as to minimize the sum of squares of the relative error between the corrected data and a quadratic fit describing the conductance as a function of the measured voltage. The choice of a value for $\lambda$ which is too low, or too high, leads to a divergence of the data from the best fit.

Fig. 5 shows an example of this procedure applied to measured calibration data for a WCT-120 photoconductance system. Calibration samples consisted of wafers with a range of resistivities

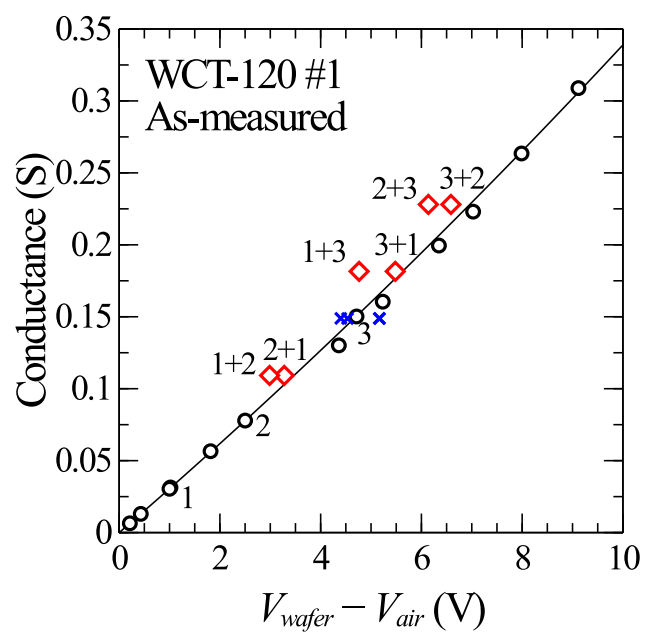

(a)

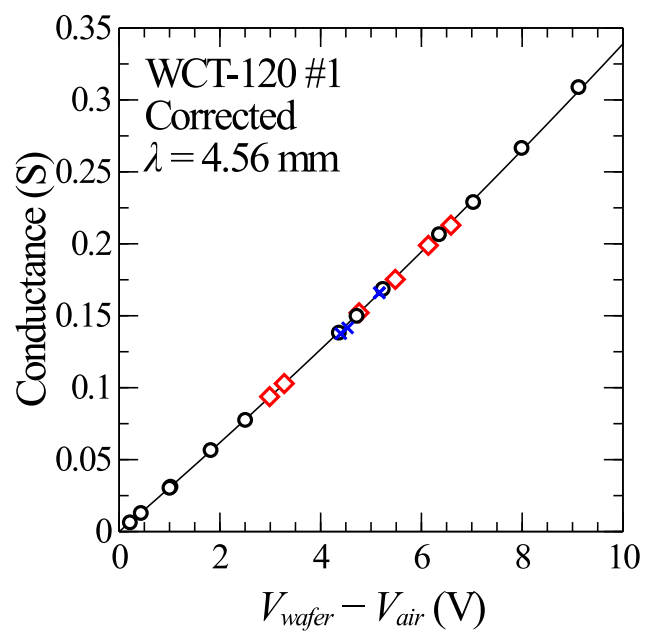

(b)

Fig. 5. (a) Sample conductance measured by four-point-probe versus coil voltage measured by WCT-120 System \#1 for silicon wafer calibration samples of various resistivity and thickness either measured singly (circles) or in stacks (diamonds), where the labels indicate the order of stacking, as well as singleside-diffused wafers (crosses) measured with the diffused side either facing toward or away from the coil. (b) Same data corrected for the relative sensitivity of the coil according to (6) with $\lambda=4.56 \mathrm{~mm}$, normalized to the sensitivity for a $525-\mu \mathrm{m}$-thick sample. In both cases the line is a quadratic fit to the final corrected data.

and thicknesses and were measured both singly and stacked in different configurations. Most of the samples had a thickness close to $525 \mu \mathrm{m}$. Because the relative sensitivity is higher for material closer to the coil, a larger voltage is measured for thinner wafers of a given conductance, and a smaller voltage is measured for stacked wafers than would be expected from the sum of their conductances [see Fig. 5(a)]. Furthermore, because the conductance of the stacked wafers is different, the measured voltage depends on the order in which they are stacked. We also measured single-side-diffused wafers with the same sheet resistance but two different thicknesses, both with the diffused side facing toward the coil and away from it. For these samples, a lower voltage is measured when the diffused side is facing away from the coil, because the coil is less sensitive 
TABLE I

Summary of Modeled and EXPERIMENTAl ATtENuATION LENGTHS FOR DifFERENT PHOTOCONDUCTANCE Systems

\begin{tabular}{|c|c|c|c|}
\hline System & $\begin{array}{l}\text { Calibration } \\
\text { Sample Set }\end{array}$ & $\lambda(\mathbf{m m})$ & $\begin{array}{l}\text { \% Deviation } \\
\text { from Model }\end{array}$ \\
\hline WCT-120 $\left(\varnothing_{\text {coil }}=18.74 \mathrm{~mm}, \varnothing_{\text {plate }}=\mathbf{4 0} \mathrm{mm}\right)$ & & 4.22 (Modeled) & \\
\hline \#1 (ANU) & 2 & 4.56 & $8.1 \%$ \\
\hline \#2 (Fraunhofer ISE) & 2 & 4.66 & $10.4 \%$ \\
\hline \#3 (Fraunhofer ISE) & 2 & 4.78 & $13.3 \%$ \\
\hline WCT-120PL $\left(\varnothing_{\text {coil }}=\mathbf{1 8 . 7 4} \mathbf{m m}, \varnothing_{\text {plate }}=\mathbf{4 0} \mathbf{m m}\right)$ & & 4.22 (Modeled) & \\
\hline$\# 1(\mathrm{ANU})$ & 2 & 4.47 & $5.9 \%$ \\
\hline \#2 (Warwick) & 2 & 4.59 & $8.8 \%$ \\
\hline WCT-120TS $\left(\varnothing_{\text {coil }}=16.8 \mathrm{~mm}, \varnothing_{\text {plate }}=30 \mathrm{~mm}\right)$ & & 3.46 (Modeled) & \\
\hline \#1 (Eindhoven) & 1 & 3.22 & $-6.9 \%$ \\
\hline \#1 (Eindhoven) & 2 & 3.33 & $-3.7 \%$ \\
\hline WCT Custom $\left(\varnothing_{\text {coil }}=9 \mathrm{~mm}, \varnothing_{\text {plate }}=\mathbf{1 6} \mathrm{mm}\right)$ & & 1.93 (Modeled) & \\
\hline \#1 (Warwick) & 2 & 2.05 & $6.2 \%$ \\
\hline
\end{tabular}

ANU $=$ The Australian National University, Eindhoven $=$ Eindhoven University of Technology, Fraunhofer ISE $=$ Fraunhofer Institute for Solar Energy Systems, Warwick = Warwick University

to the highly conductive diffused region when it is further away.

Fig. 5(b) shows the same data after correcting the conductance to account for the relative sensitivity of the coil using (2), (3), and (6). $\Delta z$ is assumed to be equal to zero for wafers placed directly on the measurement stage and equal to the thickness of the bottom wafer for the top wafer in a stack. In this example, we have chosen to divide $\sigma_{\text {eff }}$ for all samples by $S(W=525 \mu \mathrm{m})$ (the approximate thickness of eight of the 13 bulk-doped samples), in order to emphasize the relative correction applied to the thinner samples and stacks (the resulting calibration curve would, therefore, be applicable to $525-\mu$ m-thick samples). Excellent convergence of the data is achieved for $\lambda=4.56 \mathrm{~mm}$, with a relative error of less than $1 \%$ compared to the quadratic fit for all but one data point. This is within $11 \%$ of the value of $\lambda=4.22 \mathrm{~mm}$ predicted by modeling. We conservatively estimate the uncertainty in $\lambda$ determined in this way to be approximately $\pm 0.50 \mathrm{~mm}$, or $11 \%$ relative. This is based on assumed uncertainties of $\pm 1^{\circ} \mathrm{C}$ in temperature, $\pm 3 \mu \mathrm{m}$ in sample thickness, $1 \%$ relative error in the measured coil voltage, and an uncertainty in the conductance given by two standard deviations of the values measured at multiple points across each sample.

The same procedure was applied to similar measurements performed on a number of individual photoconductance lifetime measurement systems in several different laboratories. Table I summarizes the resulting attenuation lengths and compares them to the modeled values for each system. In general, there is good agreement between the experimental and modeled values. In particular, the experimental values for the WCT-120TS and WCT Custom systems are quite close to the modeled values, while the experimental WCT-120 and WCT-120PL values tend to be slightly higher. This is reasonable given the fact that the latter systems feature a layer of plastic casing between the coil and the sample, while in the former the coil cavity is uncovered and the sample sits in close proximity to the coil. In the modeling, the gap between the coil and the sample was assumed to be only $10 \mu \mathrm{m}$. A larger gap, such as that resulting from any casing, would be expected to increase the apparent attenuation coefficient. While some system-to-system variation might be expected given likely manufacturing tolerances, the observed variations among the examined systems appear to be within the measurement uncertainty.

Most systems were measured using calibration sample set \#2. In order to assess the extent of possible systematic errors in the value of $\lambda$ due to the use of this particular sample set, measurements of this sample set and the independently characterized sample set\#1 were performed immediately following each other using the same system (WCT-120TS \#1). The resulting values for $\lambda$ (see Table I) agreed to within $3.2 \%$.

\section{B. Direct Measurement of the Attenuation Function}

In determining experimental values for the attenuation length in Section IV-A, we assumed the validity of the functional form of the attenuation derived from numerical modeling and expressed in (1)-(3). In order to test this assumption, we designed an experiment to measure the attenuation function directly. For this purpose, we prepared a sample consisting of a 376- $\mu \mathrm{m}$ thick high-resistivity wafer substrate with a highly conductive diffused region on one side as described in Section II, such that the relative contribution of the wafer bulk to the total sample conductance was less than $0.25 \%$. Such a sample provides a good approximation of an infinitesimally thin conductive sheet (the thickness of the diffused region is expected to be $<1 \mu \mathrm{m}$ ).

The dark conductance of this sample was measured using WCT-120 System \#1 as a function of distance from the coil (lift-off) between 0 and $1 \mathrm{~mm}$ using 2 in square, optically polished fused silica slides with a range of thicknesses, either singly or arranged in stacks, as nonconductive spacers between the coil and the sample. Additional measurements between 1 and $10 \mathrm{~mm}$ were performed using spacer stacks consisting of $\sim 5 \mathrm{~cm}$ square pieces cut from a $\sim 1$-mm-thick polymer sheet. The thickness of each spacer was determined in the same way as for the wafer samples. For the measurements between 0 and $1 \mathrm{~mm}$, the sample was placed with the diffused side closest to 


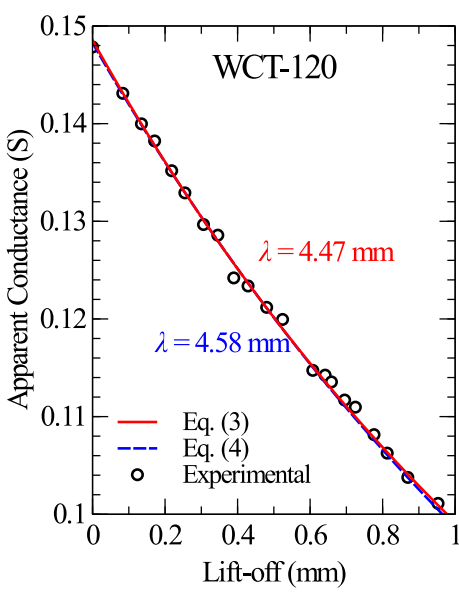

(a)

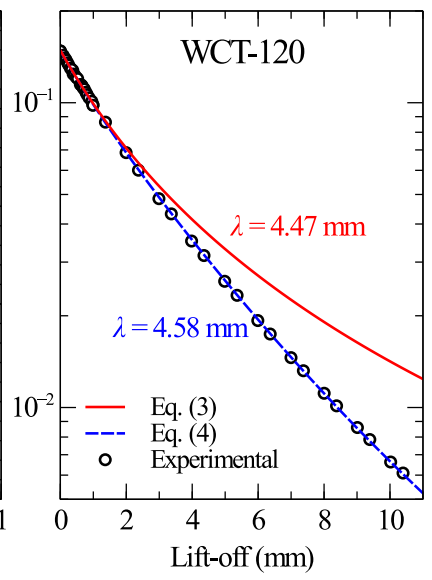

(b)
Fig. 6. Apparent sample sheet conductance measured by WCT-120 System $\# 1$ as a function of sample lift-off for a single-side-diffused wafer with a high-resistivity bulk (i.e., representative of a thin conductive sheet) for lift-off, (a) between 0 and $1 \mathrm{~mm}$, and (b) between 0 and $10 \mathrm{~mm}$. Lines show least squares fits using (3) for data between 0 and $1 \mathrm{~mm}$, and using (4) for data between 0 and $10 \mathrm{~mm}$, which yield $\lambda=4.47 \mathrm{~mm}$ and $\lambda=4.58 \mathrm{~mm}$, respectively.

the coil, while for the measurements between 1 and $10 \mathrm{~mm}$, the sample was measured in both orientations. The measured voltage was converted to apparent sheet conductance using the quadratic relationship determined for the same system from the corrected calibration measurements described in the previous section (this is necessary to account for the slight nonlinearity of the conductance-voltage relationship; however, it has only a fairly minor effect on the resulting value of $\lambda$ ).

Fig. 6 shows the resulting apparent conductance measured as a function of sample lift-off. Close to the coil, the attenuation is well-described by (3). A value of $\lambda=4.47 \mathrm{~mm}$ is derived by fitting (3) to the data between 0.1 and $1 \mathrm{~mm}$ in good agreement with the value of $4.56 \mathrm{~mm}$ derived for the same system by the calibration correction method. At larger distances, between 1 and $10 \mathrm{~mm}$, the data are better described by the more complex equation (4), consistent with the behavior predicted by modeling (compare the results of Fig. 3). A fit of (4) to the data between 0 and $10 \mathrm{~mm}$ yields $\lambda=4.58 \mathrm{~mm}$.

\section{Skin Effects}

It is well known that eddy current measurements may be subject to skin effects, whereby the opposing current induced at the surface of a conductor effectively shields the penetration of the magnetic field further into the bulk. This results in a saturation of the measured voltage for very thick or very conductive samples, as observed, for example, by Swirhun et al. [7] for bulk Si samples. The effect is commonly described in terms of a characteristic "skin depth," defined as the depth at which the field strength drops to $1 / e$.

Commonly cited formulas for the skin effect are based on the assumption of a spatially uniform magnetic field. In this case, the field strength decays exponentially with distance from the sample surface, and the skin depth is inversely proportional to the square root of the sample conductivity. However, in our

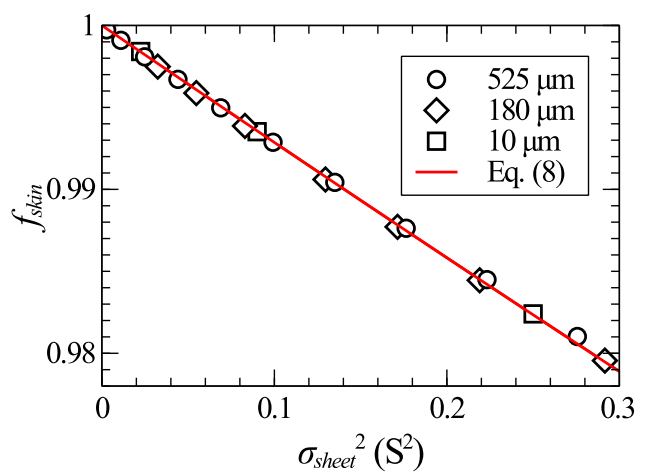

Fig. 7. Skin effect factor $f_{\text {skin }}$ as a function of the square of the sample sheet conductance $\sigma_{\text {sheet }}$ for several fixed sample thicknesses, determined from numerical modeling of the WCT-120 coil.

case, the magnetic field is spatially highly nonuniform, so that this assumption is not valid.

In order to evaluate the influence of the skin effect on our measurements, numerical simulations were performed to determine relative coil sensitivity as a function of sample conductivity at several fixed sample thicknesses for the WCT-120 coil geometry. A skin factor $f_{\text {skin }}$ was defined as the ratio of the relative sensitivity to that at near-zero conductance. As shown in Fig. 7, we found that regardless of the sample thickness, $f_{\text {skin }}$ could be described simply as an inverse linear function of the square of the sample sheet conductance $\sigma_{\text {sheet }}$, such that

$$
f_{\text {skin }}\left(\sigma_{\text {sheet }}\right)=\left(1+\sigma_{\text {sheet }}^{2} / \sigma_{\text {ref,skin }}^{2}\right)^{-1}
$$

where $\sigma_{\text {ref,skin }}$ is a constant reference conductance equal to $3.73 \mathrm{~S}$

The most conductive sample in our calibration set had a sheet conductance of $0.318 \mathrm{~S}$. This corresponds to $f_{\text {skin }}=0.9928$, or a $0.72 \%$ reduction in apparent conductance according to (8). The effect is therefore small, if not entirely negligible. Since the general effect of (8) is simply to introduce a slight nonlinear component into the relationship between coil voltage and sample conductance, we consider for our purposes that this effect is effectively absorbed within the quadratic fit applied to the conductance versus voltage calibration data (see Fig. 5). It should be noted that the magnitude of the effect predicted by (8) can only account for a minor part of the nonlinearity invariably observed experimentally in such calibration curves.

\section{Consequences for Lifetime Measurements}

Failure to account for the dependence of coil sensitivity on sample thickness and lift-off will result in a systematic relative error in the measured sheet conductance $\sigma_{\text {sheet }}$ when sample thickness or lift-off is different from that of the samples used in calibration. This will translate into errors in the injectiondependent excess carrier lifetime $\tau_{\text {eff }}(\Delta n)$ determined from photoconductance measurements.

For a uniformly conductive sample of thickness $W$, measured by a coil calibrated using uniformly doped wafers of thickness $W_{\text {cal }}$, the relative error $\delta\left(\sigma_{\text {sheet }}\right)$ in the measured sheet conductance due to the coil sensitivity attenuation is independent of the 
sample conductance and simply given by

$$
\delta\left(\sigma_{\text {sheet }}\right)=\frac{S(W, \Delta z)}{S\left(W_{\text {cal }}, \Delta z_{\text {cal }}\right)}-1
$$

where $\Delta z$ and $\Delta z_{\text {cal }}$ are the lift-off of the sample and the calibration wafers, respectively.

The standard calibration wafers supplied by Sinton Instruments have a thickness of $525 \mu \mathrm{m}$, while a common sample thickness is $180 \mu \mathrm{m}$. Taking the value of $\lambda=4.56 \mathrm{~mm}$ measured for system WCT-120 \#1, this corresponds to a relative error $\delta\left(\sigma_{\text {sheet }}\right)=7.3 \%$ in the measured conductance from (2) and (9) with $\Delta z=\Delta z_{\mathrm{cal}}=0$.

In photoconductance lifetime measurements, the excess carrier concentration $\Delta n$ as a function of time $t$ is calculated from the excess (nonequilibrium) component of the sheet conductance $\Delta \sigma_{\text {sheet }}$ according to [1], [2], [4]

$$
\Delta n(t)=\frac{\Delta \sigma_{\text {sheet }}(t)}{q \mu_{\text {sum }}(\Delta n) W}
$$

where $q$ is the elementary charge, and $\mu_{\text {sum }}=\mu_{e}+\mu_{h}$ is the sum of the electron and hole mobilities. We write $\mu_{\text {sum }}(\Delta n)$ to indicate explicitly that the latter is a function of $\Delta n$ [consequently (10) is an implicit equation and must generally be solved iteratively].

For small $\delta\left(\sigma_{\text {sheet }}\right)$, the relative error in $\Delta n(t)$ may be described by the first-order approximation

$$
\delta(\Delta n(t)) \approx \delta\left(\sigma_{\text {sheet }}\right)\left(1+\frac{\Delta n}{\mu_{\text {sum }}} \frac{\mathrm{d} \mu_{\text {sum }}}{\mathrm{d} \Delta n}\right)^{-1} .
$$

From (11), it is apparent that a given error in $\sigma_{\text {sheet }}$ will result in a somewhat larger error in $\Delta n(t)$ due to the fact that $\mu_{\text {sum }}$ also decreases with increasing $\Delta n$ (i.e., $\left.\mathrm{d} / \mathrm{d} \Delta n\left(\mu_{\text {sum }}\right)<0\right)$. Because the relative rate of decrease of $\mu_{\text {sum }}$ [represented by the second term in (11)] increases with increasing $\Delta n$ in the range covered by typical photoconductance measurements, this error will also increase with $\Delta n$.

In the general case, the effective excess carrier lifetime $\tau_{\text {eff }}$ is calculated from $\Delta n(t)$ according to [5]

$$
\tau_{\text {eff }}(t)=\Delta n\left(G(t)-\frac{\mathrm{d} \Delta n}{\mathrm{~d} t}\right)^{-1}
$$

where $G(t)$ is the time-dependent generation rate due to optical excitation. Under transient conditions, $G=0$, and the error in $\Delta n(t)$ mostly cancels with the error in $\mathrm{d} \Delta n / \mathrm{d} t$. In this case, the value of $\tau_{\text {eff }}(t)$ is essentially correct, and only $\Delta n(t)$ is in error. However, because in general $\tau_{\text {eff }}$ is a function of $\Delta n$, the error in $\Delta n(t)$ will likely still result in an error in $\tau_{\text {eff }}(\Delta n)$. When $\tau_{\text {eff }}$ is strongly dependent on $\Delta n$, as, for example, under high injection conditions, this error may be substantial. Under quasi-steady-state (QSS) conditions, $\mathrm{d} \Delta n / \mathrm{d} t=0$, and the error in $\Delta n(t)$ results in an additional identical error in $\tau_{\text {eff }}(t)$ (i.e., $\left.\delta\left(\tau_{\text {eff }}(t)\right)=\delta(\Delta n(t))\right)$, which compounds the overall error in $\tau_{\text {eff }}(\Delta n)$. Excitation conditions intermediate between the transient and QSS extremes will result in correspondingly intermediate errors.

We will now give an experimental example to illustrate the sort of errors that may be expected more concretely.

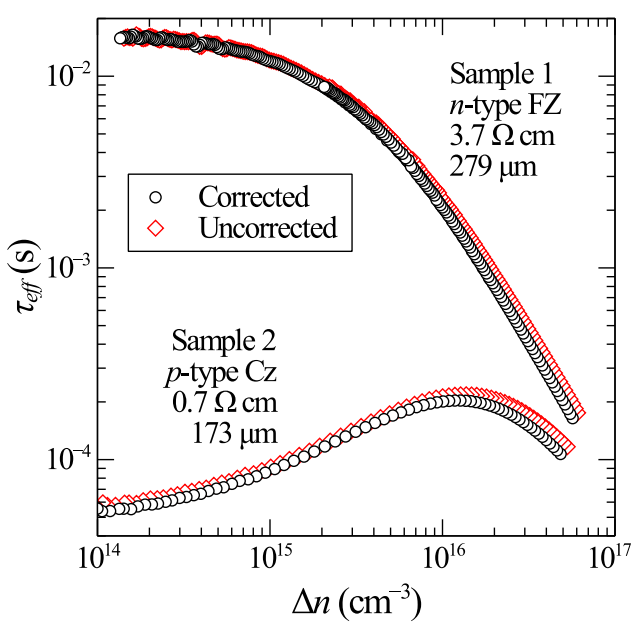

Fig. 8. Experimental carrier lifetime $\tau_{\text {eff }}$ as a function of excess carrier concentration $\Delta n$, measured for two samples by WCT-120 System \#1. Data are shown both uncorrected for the effect of sample thickness on coil sensitivity (using the calibration coefficients determined for 525- $\mu$ m-thick samples) and corrected for this effect.

Fig. 8 shows $\tau_{\text {eff }}(\Delta n)$ measured for two silicon wafer samples using WCT-120 System \#1, where the calibration was corrected for sample thickness as described in this article. The first sample is a $3.7 \Omega \mathrm{cm}$ n-type FZ wafer with a thickness of $279 \mu \mathrm{m}$. It is well-passivated, with an effective lifetime of over $10 \mathrm{~ms}$ in low injection. This sample is representative of the highlifetime samples which are the subject of surface passivation and intrinsic bulk lifetime studies. The second sample is a $0.7 \Omega \mathrm{cm}$ p-type $\mathrm{Cz}$ wafer with a thickness of $173 \mu \mathrm{m}$. It is moderately well-passivated, with a peak lifetime around $200 \mu \mathrm{s}$ at $\Delta n=\sim 1 \times 10^{16} \mathrm{~cm}^{-3}$, but has a certain amount of extrinsic bulk recombination that reduces the lifetime in low injection. This sample is representative of industrial solar cell precursors.

Fig. 8 also shows the apparent $\tau_{\text {eff }}(\Delta n)$ that would be measured for each sample if the calibration were not corrected for sample thickness, assuming calibration were performed using $525-\mu \mathrm{m}$-thick samples. The corresponding error in the measured sample conductance is $5.1 \%$ for Sample 1, and $7.4 \%$ for Sample 2, based on (9) with $S(W, \Delta z=0)$ given by (2). This clearly translates into a significant overestimation of $\tau_{\text {eff }}(\Delta n)$, especially at higher $\Delta n$.

Fig. 9 plots the resulting errors in $\Delta n(t), \tau_{\text {eff }}(t)$, and $\tau_{\text {eff }}(\Delta n)$ as a function of $\Delta n$ for each sample, when excited using either a short "transient" flash (1/64 power) or an extended "QSS" flash (1/1 power). Note that while we will refer to the latter excitation conditions as QSS, true QSS conditions do not necessarily obtain in both samples throughout this measurement, as we shall discuss. The error in $\Delta n(t)$ is independent of the excitation conditions and is simply equal to the error in the conductance at low $\Delta n$. It increases with $\Delta n$ in accordance with (11). The error in $\tau_{\text {eff }}(t)$ is negligible under transient excitation conditions, but approaches the error in $\Delta n(t)$ under QSS excitation conditions. For Sample 1 excited by the QSS flash, the error in $\tau_{\text {eff }}(t)$ shows a transition from true QSS conditions $\left(\delta\left(\tau_{\text {eff }}(t)\right) \approx \delta(\Delta n(t))\right)$ at high $\Delta n$, to an intermediate regime where $G(t)$ and $\mathrm{d} \Delta n / \mathrm{d} t$ 


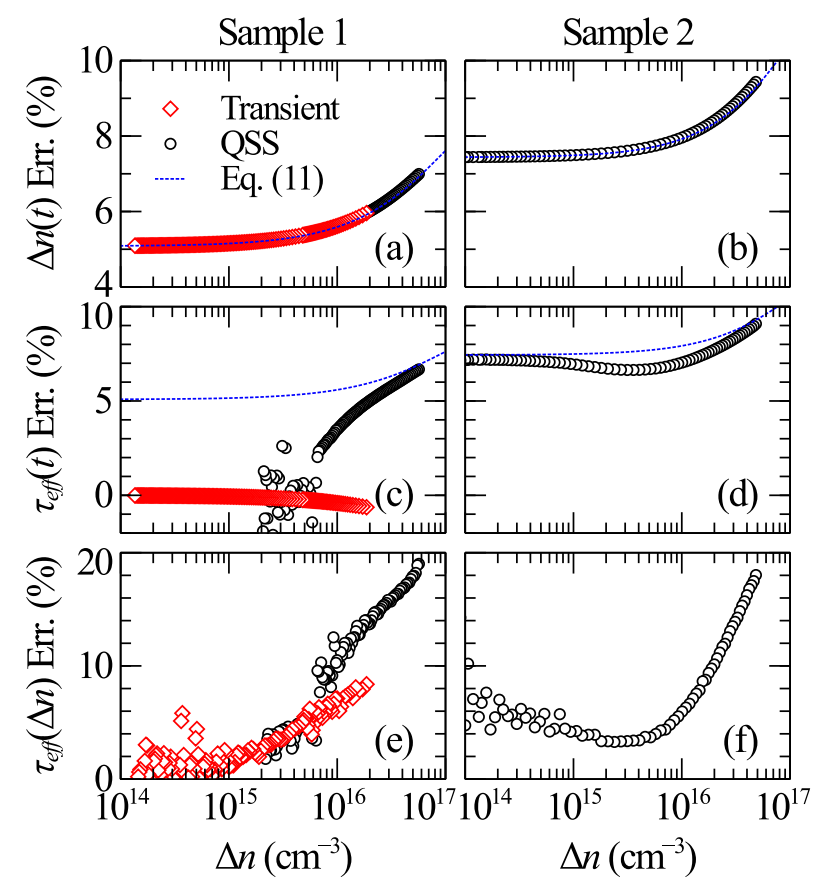

Fig. 9. Relative error in (a), (b) $\Delta n(t)$, (c), (d) $\tau_{\text {eff }}(t)$, and (e), (f) $\tau_{\text {eff }}(\Delta n)$ as a function of $\Delta n$ for the uncorrected lifetime curves shown in Fig. 8 for Samples 1 and 2, compared to the corrected values. Data are shown for measurements using both "transient" and "QSS" flashes for Sample 1, and "QSS" flash only for Sample 2. Dotted lines show the first order approximation to $\delta(\Delta n(t))$ given by (11).

are of comparable magnitude, and $0<\delta\left(\tau_{\text {eff }}(t)\right)<\delta(\Delta n(t))$. This occurs because the exponential decay lifetime of the QSS flash excitation $(\sim 2 \mathrm{~ms})$ is initially significantly longer than $\tau_{\text {eff }}$ of this sample at high $\Delta n$, but becomes shorter than $\tau_{\text {eff }}$ as $\Delta n$ decreases.

The errors in $\Delta n(t)$ and $\tau_{\text {eff }}(t)$ are compounded into more significant errors in $\tau_{\text {eff }}(\Delta n)$ due to the strong dependence of $\tau_{\text {eff }}$ on $\Delta n$ (see Fig. 8). This is especially the case for Sample 1 , since for this sample $\tau_{\text {eff }}^{-1}$ approaches an approximate square dependence on $\Delta n$ (limited by Auger recombination) in high injection, while for Sample 2, which is much more heavily doped and also limited partly by extrinsic bulk and surface recombination, the dependence of $\tau_{\text {eff }}^{-1}$ on $\Delta n$ at the highest injection levels is never more than linear. As a result, even though $\delta\left(\sigma_{\text {sheet }}\right)$ is greater for the thinner Sample 2, the error in $\tau_{\text {eff }}(\Delta n)$ [see Fig. 9(e) and (f)] is greater for Sample 1 over most of the injection range when excited by the longer "QSS" flash. It decreases sharply with decreasing $\Delta n$ mainly because of the reduction in $\delta\left(\tau_{\text {eff }}(t)\right)$ [see Fig. 9(c)] due to the aforementioned transition from true QSS conditions to an intermediate regime. The net result is a strongly injection-dependent error in $\tau_{\text {eff }}(\Delta n)$, rising from $\sim 11 \%$ at $\Delta n=1 \times 10^{16} \mathrm{~cm}^{-3}$ to $\sim 17 \%$ at $\Delta n=4 \times 10^{16} \mathrm{~cm}^{-3}$. The error in $\tau_{\text {eff }}(\Delta n)$ for Sample 2 increases from $\sim 6 \%$ to $\sim 16.5 \%$ in the same range. The error in the data measured under transient excitation is somewhat smaller, because in this case the contribution from $\delta\left(\tau_{\text {eff }}(t)\right)$ is negligible, but still rises to $\sim 7 \%$ at $\Delta n=1 \times 10^{16} \mathrm{~cm}^{-3}$. In the injection range below $\sim 1 \times 10^{16} \mathrm{~cm}^{-3}$, the error in $\tau_{\text {eff }}(\Delta n)$ for Sample 2 is actually reduced below $\delta\left(\tau_{\mathrm{eff}}(t)\right)$, because in this range the lifetime increases with $\Delta n$, so that the errors in $\Delta n(t)$ and $\tau_{\text {eff }}(t)$ partially compensate each other.

Such errors in $\tau_{\text {eff }}(\Delta n)$ are significant for a range of applications. We will mention three here: 1) $J_{0}$ extraction via the method of Kane and Swanson [1],2) determination of the surface recombination velocity and bulk lifetime from wafer thickness variation experiments, and 3) determination of the injectiondependent Auger recombination rate. Let us now address each of these in turn.

When measuring the surface saturation current density $J_{0}$ via the method of Kane and Swanson [1], it is necessary to subtract the intrinsic bulk component (mainly due to Auger recombination) from the total measured recombination rate. This is calculated based on a suitable model using the measured value of $\Delta n$. Since Auger recombination is approximately proportional to the square of $\Delta n$ in high injection, a relative error $\delta(\Delta n)$ in $\Delta n$ leads to an error of roughly $(\delta(\Delta n))^{2}$ in the calculated Auger recombination rate. This results in an absolute error in $J_{0}$ on the order of several $\mathrm{fA} \mathrm{cm}^{-2}$. Given that technologically achievable $J_{0}$ values are themselves now in the range of a few $\mathrm{fA} \mathrm{cm}^{-2}$, this can represent a significant relative error. This error occurs in addition to a relative error component due to the error in $\tau_{\text {eff }}(\Delta n)$. The absolute error will dominate the relative error when $J_{0}$ is very small, and vice versa when $J_{0}$ is large.

An established method of separating the contributions of bulk and surface recombination processes without the need to make assumptions regarding the bulk lifetime is to measure $\tau_{\text {eff }}(\Delta n)$ for a set of otherwise identical samples with a range of thicknesses [17], [18]. The effective surface recombination velocity $S_{\text {eff }}$ at a given value of $\Delta n$ may then be determined from the slope of a linear fit to $\tau_{\text {eff }}^{-1}$ versus inverse thickness, while the inverse bulk lifetime $\tau_{\text {bulk }}^{-1}$ is given by the intercept of the same plot. The dependence of coil sensitivity on sample thickness described in this article presents obvious problems for this method. Specifically, if thickness is not accounted for, $\tau_{\text {eff }}(\Delta n)$ of the thinner samples will be overestimated relative to that of the thicker samples. This will result in a systematic underestimation of both $S_{\text {eff }}$ and $\tau_{\text {bulk. }}$.

Finally, in experiments intended to determine the intrinsic Auger lifetime, such as those of [18]-[21], a given relative error in $\tau_{\text {eff }}(\Delta n)$ would be expected to lead to an identical error in the extracted Auger lifetime. Fig. 9(e) and (f) shows that this will particularly affect lifetime values in high injection. Where that error is a function of $\Delta n$, as in Fig. 9(e), this could also lead to an error in the exponent attributed to the $\Delta n$ dependence of the Auger recombination.

\section{E. Radial Sensitivity}

As well as the dependence on the distance, the lateral spatial variation of the coil sensitivity is also important in some applications, particularly in correlating spatially resolved lifetime measurements (from, e.g., photoluminescence imaging) with inductively coupled photoconductance measurements of samples with spatially nonuniform lifetime, e.g., such as those based on multicrystalline Si [22], [23]. Given the radial symmetry 


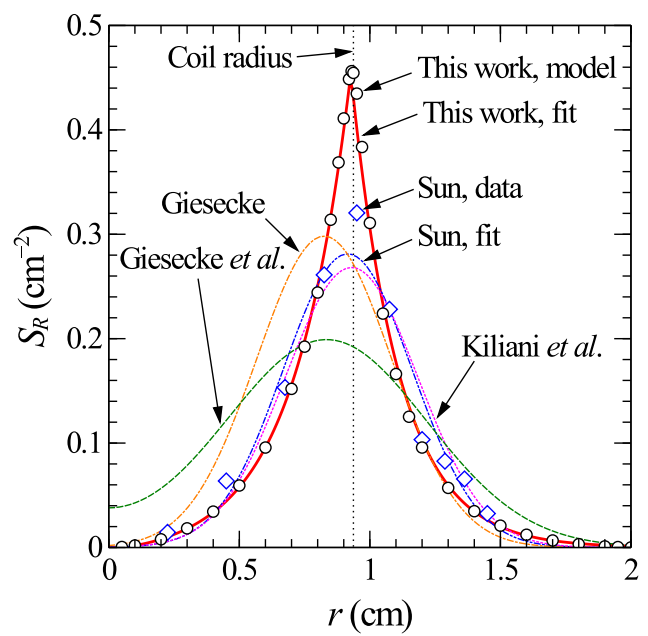

Fig. 10. Radial sensitivity $S_{R}$ of the WCT-120 system as a function of radial distance $r$ measured from the center of the coil. Circles show numerically modeled values from this article, while the continuous solid line shows the fit of these values using (14) with the parameters given by Table II. Also shown are the radial sensitivity functions reported by various authors [22]-[25] on the basis of various experimental approaches, which in all cases assumed a Gaussian distribution. The experimental sensitivity derived by direct numerical differentiation of the experimental data of [25] is also shown.

of the coil, the lateral sensitivity may be described simply by a radial sensitivity function $S_{R}(r)$ defined for radius $r>=0$, where $r=0$ at the coil axis. Several authors have attempted to determine the radial sensitivity function experimentally using various experimental approaches [22]-[25]. All have assumed that $S_{R}(r)$ can be described by a Gaussian function. However, this assumption has not been physically justified or rigorously tested.

The numerical model of the coil impedance, which we have developed and validated for the case of varying sample thickness and lift-off, can also be readily applied to assess the radial sensitivity variation. Fig. 10 shows the modeled relative sensitivity of the WCT-120 system as a function of radial distance $r$ from the center of the coil, which was determined by simulating narrow ( $10 \mu \mathrm{m}$ wide) radial cross sections of a $180-\mu \mathrm{m}$-thick sample centerd at various values of $r$. This approach is valid because a radial symmetry requires that all current flows radially (the same dependence resulted from numerical differentiation of the normalized sensitivity modeled for samples of varying radius). The resulting change in impedance was normalized to the value modeled for a $10 \mathrm{~cm}$ diameter (i.e., effectively infinite) sample of the same thickness, and divided by $2 \pi r$ to give the radial sensitivity. We define the magnitude of the radial sensitivity $S_{R}$ such that

$$
\int_{0}^{\infty} 2 \pi r S_{R} \mathrm{~d} r=1
$$

$S_{R}$, therefore, has units of inverse area. We consider this approach preferable to that of [22], which contrived to define $S_{R}$ as a unitless quantity by normalizing it to an arbitrary area.

Fig. 10 shows that the modeled radial sensitivity exhibits a peak at $r$ close to the radius of the coil, in agreement with previous experimental results, especially those of [23] and [25].
TABLE II

Parameter VAlues of (14) FOR THE Fit OF Fig. 10.

\begin{tabular}{cccccc}
\hline Parameter & Value & Parameter & Value & Parameter & Value \\
\hline$A$ & 0.48 & $b_{1}(\mathrm{~mm})$ & 1.99 & $b_{3}(\mathrm{~mm})$ & 2.21 \\
$r_{0}(\mathrm{~mm})$ & 9.27 & $b_{2}(\mathrm{~mm})$ & 20.3 & & \\
\hline
\end{tabular}

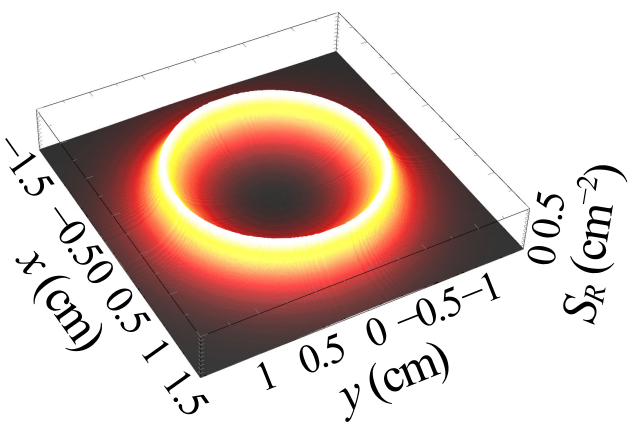

Fig. 11. 3-D surface representation of the spatial sensitivity of the WCT-120 coil. The sensitivity is highest directly above the wires of the coil, and drops off sharply with distance. At the center of the coil and beyond the edge of the field plate it is essentially zero.

However, the peak is markedly sharper than a Gaussian function. Instead it is better described as a slightly asymmetric, doublesided exponential decay centered on the coil windings, which tends to zero at the center of the coil and at the edge of the field plate. The following function, combined with the parameter values in Table II, provides a good description of the modeled data, as shown in Fig. 10:

$$
\begin{aligned}
& S_{R}(r) \\
& =A \exp \left(-\frac{\left|r-r_{0}\right|}{b_{1}}\right) \exp \left(-\frac{r-r_{0}}{b_{2}}\right)\left(\frac{r^{2}}{b_{3}^{2}+r^{2}}\right) .
\end{aligned}
$$

Here the second term accounts for skewness, while the final term enforces the physical requirement that the sensitivity go to zero at $r=0$. Fig. 11 shows a 3-D surface representation of the corresponding spatial sensitivity function. The sensitivity is highest opposite the coil windings, and drops off sharply with distance. At the center of the coil it is zero.

Direct comparison with experimental data for $S_{R}(r)$ is not straightforward because in most previous work $S_{R}(r)$ was not measured directly but only in a somewhat convoluted form. The measurements of [22] and [23] were performed under nonequilibrium conditions and were, therefore, subject to carrier diffusion broadening, while analysis of the data of [24] is complicated by the influence of unknown geometric factors necessary to account for incomplete radial current flow paths. The experiment of Sun [25], which was performed by measuring the relative dark conductance of identically doped wafers laser-cut to various radii, involves the least complications of interpretation. While Sun chose to fit the data using a Gaussian function for $S_{R}(r)$, the data are also susceptible to direct interpretation via simple numerical differentiation. In Fig. 10, we also plot $S_{R}(r)$ derived from the data of Sun by numerical differentiation using the midpoint slope method. Despite the limited resolution of the data there is clear evidence of a sharper peak than described by 


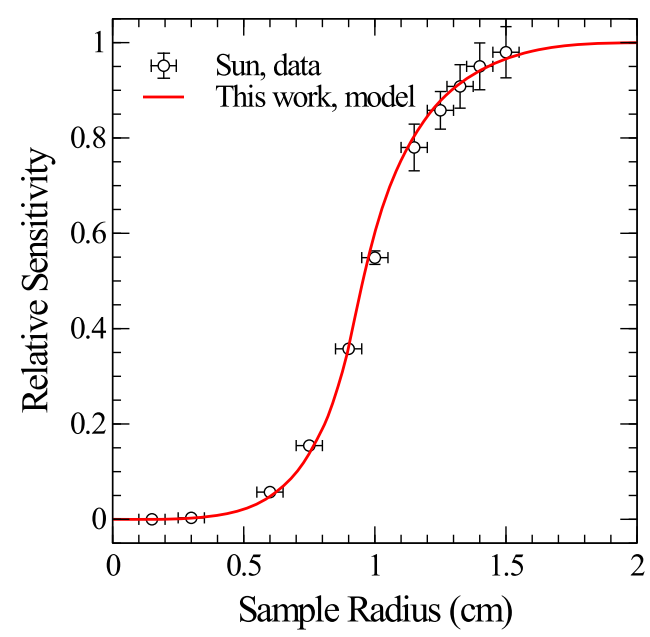

Fig. 12. Relative sensitivity as a function of sample radius for samples centered on the coil axis. Data points show the experimental measurements of [25], which were derived from dark conductance measurements of identically doped wafers laser-cut to various diameters, using the WCT-120 system. Vertical error bars show the $2 \sigma$ error derived from measurements of multiple samples at each diameter, while horizontal error bars account in an approximate way for an assumed $\pm 0.5 \mathrm{~mm}$ alignment error with respect to the coil. The line shows the dependence predicted by numerical modeling of the same system.

the Gaussian fit. We note that any alignment error of the samples relative to the center of the coil during the measurements would have the effect of broadening the apparent $S_{R}(r)$. Fig. 12 plots the original experimental data of [25] for relative conductance as a function of sample radius, compared to the dependence predicted by our numerical model. There is excellent agreement within the range of experimental uncertainty.

\section{CONCLUSION}

We have shown that the relative sensitivity of the inductive coil used to measure the sample conductance in widely used photoconductance lifetime measurement systems depends significantly on sample thickness (as well as lift-off) in the range of typical silicon wafer thicknesses. Simple empirical expressions describing the dependence of the sensitivity on sample thickness and lift-off within the regime of interest were derived from numerical modeling and confirmed experimentally. The systemdependent attenuation lengths involved in these expressions were determined experimentally for various photoconductance measurement systems. These expressions may be used to correct photoconductance measurements for the effect of sample thickness and lift-off, thus eliminating a significant source of systematic error in carrier lifetimes derived from such measurements. Among other things, this will be critical to enable future high-accuracy measurements of surface and bulk recombination parameters, especially in high injection. Finally, we also applied numerical modeling to predict the radial variation of the coil sensitivity, finding good qualitative agreement with the results of some authors, but with a sharper distribution than previously assumed.

\section{ACKNOWLEDGMENT}

The authors would like to thank R. A. Sinton for valuable discussions and feedback, and for supplying the details of the coil geometry for the different photoconductance measurement systems. Additional assistance was provided by C. Sainsbury at Sinton Instruments. Initial work was performed by L. E. Black at Eindhoven University of Technology and benefited from the advice and assistance of V. Vandalon, B. van de Loo, B. Macco, J. Melskens, and E. Kessels. T. Niewelt, M. Schubert, F. Schindler, A. Richter, H. Höffler, and B. Steinhauser at Fraunhofer ISE facilitated access to their measurement systems and were helpful and hospitable, as were N. Grant and A. Pointon at Warwick University. D. Skorka is thanked for for suggesting the addition of the radial sensitivity modeling. C. Samundsett and S. Armand at ANU assisted with sample preparation, while P. Phang provided lifetime samples. C. Sun generously shared unpublished data from his Ph.D. thesis.

\section{REFERENCES}

[1] D. E. Kane and R. M. Swanson, "Measurement of the emitter saturation current by a contactless photoconductivity decay method," in Proc. Conf. Rec. 18th IEEE Photovolt. Specialists Conf., Las Vegas, NV, USA, Oct. 1985 , pp. 578-583.

[2] R. A. Sinton and A. Cuevas, "Contactless determination of currentvoltage characteristics and minority-carrier lifetimes in semiconductors from quasi-steady-state photoconductance data," Appl. Phys. Lett., vol. 69, no. 17, pp. 2510-2512, 1996.

[3] R. A. Sinton, A. Cuevas, and M. Stuckings, "Quasi-steady-state photoconductance, a new method for solar cell material and device characterization," in Proc. Conf. Rec. 25th IEEE Photovolt. Specialists Conf., May 1996, pp. $457-460$.

[4] A. Cuevas and R. A. Sinton, "Prediction of the open-circuit voltage of solar cells from the steady-state photoconductance," Prog. Photovolt., Res. Appl., vol. 5, no. 2, pp. 79-90, 1997.

[5] H. Nagel, C. Berge, and A. G. Aberle, "Generalized analysis of quasisteady-state and quasi-transient measurements of carrier lifetimes in semiconductors," J. Appl. Phys., vol. 86, no. 11, pp. 6218-6221, 1999.

[6] N. E. Grant, K. R. McIntosh, and J. T. Tan, "Evaluation of the bulk lifetime of silicon wafers by immersion in hydrofluoric acid and illumination," ECS J. Solid State Sci. Technol., vol. 1, no. 2, pp. P55-P61, 2012.

[7] J. S. Swirhun, R. A. Sinton, M. K. Forsyth, and T. Mankad, "Contactless measurement of minority carrier lifetime in silicon ingots and bricks," Prog. Photovolt., Res. Appl., vol. 19, no. 3, pp. 313-319, 2011.

[8] W. Favre et al., "Coil-to-sample distance influence on contactless QSSPC effective lifetime measurements: Application to silicon wafers passivated by thin amorphous layers," in Proc. 26th Eur. Photovolt. Solar Energy Conf., 2011, pp. 1563-1568.

[9] L. E. Black and E. Kessels, "Dependence of coil sensitivity on sample thickness in inductively coupled photoconductance measurements," in Proc. AIP Conf., 2019, vol. 2147, no. 1, Art. no. 020002.

[10] R. A. Weller, "An algorithm for computing linear four-point probe thickness correction factors," Rev. Scientific Instrum., vol. 72, no. 9, pp. 3580 3586, 2001.

[11] D. K. Schroder, Semiconductor Material and Device Characterization, 3rd ed. Hoboken, NJ, USA: Wiley, 2006.

[12] J. Albers and H. L. Berkowitz, "An alternative approach to the calculation of four-probe resistances on nonuniform structures," J. Electrochemical Soc., vol. 132, no. 10, pp. 2453-2456, 1985.

[13] D. B. M. Klaassen, "A unified mobility model for device simulation-I. Model equations and concentration dependence," Solid-State Electron. vol. 35, no. 7, pp. 953-959, 1992.

[14] D. B. M. Klaassen, "A unified mobility model for device simulationII. Temperature dependence of carrier mobility and lifetime," Solid-State Electron., vol. 35, no. 7, pp. 961-967, 1992.

[15] C. V. Dodd and W. E. Deeds, "Analytical solutions to eddy-current probecoil problems,” J. Appl. Phys., vol. 39, no. 6, pp. 2829-2838, 1968. 
[16] D. C. Meeker, Finite element method magnetics, version 4.2. Feb. 25, 2018 build). [Online]. Available: http://www.femm.info. Accessed on: Mar. 21, 2019.

[17] E. Yablonovitch, D. L. Allara, C. C. Chang, T. Gmitter, and T. B. Bright, "Unusually low surface-recombination velocity on silicon and germanium surfaces," Phys. Rev. Lett., vol. 57, pp. 249-252, Jul. 1986.

[18] A. Richter, S. W. Glunz, F. Werner, J. Schmidt, and A. Cuevas, "Improved quantitative description of Auger recombination in crystalline silicon," Phys. Rev. B, vol. 86, pp. 165202-1-165202-14, Oct. 2012.

[19] E. Yablonovitch and T. Gmitter, "Auger recombination in silicon at low carrier densities,” Appl. Phys. Lett., vol. 49, no. 10, pp. 587-589, 1986.

[20] M. J. Kerr and A. Cuevas, "General parameterization of Auger recombination in crystalline silicon," J. Appl. Phys., vol. 91, no. 4, pp. 2473-2480, 2002.

[21] B. A. Veith-Wolf, S. Schäfer, R. Brendel, and J. Schmidt, "Reassessment of intrinsic lifetime limit in $n$-type crystalline silicon and implication on maximum solar cell efficiency," Solar Energy Mater. Solar Cells, vol. 186 , pp. 194-199, 2018.
[22] J. A. Giesecke, M. C. Schubert, B. Michl, F. Schindler, and W. Warta, "Minority carrier lifetime imaging of silicon wafers calibrated by quasisteady-state photoluminescence," Solar Energy Mater. Solar Cells, vol. 95 , no. 3, pp. 1011-1018, 2011.

[23] D. Kiliani et al., "Minority charge carrier lifetime mapping of crystalline silicon wafers by time-resolved photoluminescence imaging," J. Appl. Phys., vol. 110, no. 5, 2011, Art. no. 054508.

[24] J. Giesecke, "Quantitative recombination and transport properties in silicon from dynamic luminescence,” Ph.D. dissertation, Dept. Phy., Univ. Konstanz, Konstanz, Germany, 2013.

[25] C. Sun, "Recombination activity of metal-related and boron-oxygen defects in crystalline silicon," Ph.D. dissertation, The Australian Nat. Univ., Canberra, ACT, Australia, 2017. 